\title{
Functional Magnetic Resonance Imaging
}

National Cancer Institute

\section{Source}

National Cancer Institute. Functional Magnetic Resonance Imaging. NCI Thesaurus.

Code $\mathrm{C17958.}$

The principle of functional MRI imaging is to take a series of images of an organ in quick succession and to statistically analyze the images for differences among them. Most commonly used in studies of brain function. 